OPEN ACCESS

Edited by:

Johannes Kaesmacher,

Technical University of

Munich, Germany

Reviewed by:

Hamdollah Panahpour, Ardabil University of Medical

Sciences, Iran

Steffen Tiedt

Hospital of the University of

Munich, Germany

${ }^{*}$ Correspondence:

Jincai He

hic@wmu.edu.cn

Specialty section:

This article was submitted to

Stroke,

a section of the journal

Frontiers in Neurology

Received: 19 August 2019 Accepted: 26 November 2019 Published: 10 December 2019

Citation:

He W, Ruan Y, Yuan C, Cheng Q,

Cheng $H$, Zeng $Y$, Chen $Y$, Huang $G$,

Chen $\mathrm{H}$ and $\mathrm{He} \mathrm{J}$ (2019) High

Neutrophil-to-Platelet Ratio Is

Associated With Hemorrhagic

Transformation in Patients With Acute

Ischemic Stroke.

Front. Neurol. 10:1310.

doi: 10.3389/fneur.2019.01310

\section{High Neutrophil-to-Platelet Ratio Is Associated With Hemorrhagic Transformation in Patients With Acute Ischemic Stroke}

\author{
Weilei He ${ }^{1}$, Yiting Ruan ${ }^{1}$, Chengxiang Yuan ${ }^{1}$, Qianqian Cheng ${ }^{2}$, Haoran Cheng ${ }^{1}$, \\ Yaying Zeng ${ }^{2}$, Yunbin Chen ${ }^{1}$, Guiqian Huang ${ }^{1}$, Huijun $\mathrm{Chen}^{1}$ and Jincai He ${ }^{1 *}$ \\ ${ }^{1}$ Department of Neurology, The First Affiliated Hospital of Wenzhou Medical University, Wenzhou, China, ${ }^{2}$ Department of \\ Mental Health, Mental Health School, Wenzhou Medical University, Wenzhou, China
}

Background: Hemorrhagic transformation $(H T)$ is a complication that may cause neurological deterioration in patients with acute ischemic stroke. Both neutrophil and platelet have been associated with the stroke progression. The aim of this study was to explore the relationship between neutrophil-to-platelet ratio (NPR) and HT after acute ischemic stroke.

Methods: A total of 279 stroke patients with HT were consecutively recruited. HT was diagnosed using magnetic resonance imaging (MRI) or computed tomography (CT) and classified into hemorrhagic infarction $(\mathrm{HI})$ and parenchymal hematoma $(\mathrm{PH})$. Blood samples for neutrophil and platelet counts were obtained at admission. Meanwhile, 270 age- and gender-matched controls without HT were included for comparison.

Results: Among the patients with $\mathrm{HT}, 131$ patients had $\mathrm{PH}$ and 148 patients had $\mathrm{HI}$. NPR was higher in patients with PH than those with $\mathrm{HI}$ or non-HT [36.8 (23.7-49.2) vs. 26.6 (17.9-38.3) vs. 19.1 (14.8-24.8), $P<0.001$ ]. After adjustment for potential confounders, high NPR remained independently associated with the increased risk of $\mathrm{HT}(\mathrm{OR}=2.000,95 \% \mathrm{Cl}: 1.041-3.843, P=0.037)$. NPR (>39.9) was independently associated with $\mathrm{PH}(\mathrm{OR}=2.641,95 \% \mathrm{Cl}: 1.308-5.342, P=0.007)$.

Conclusions: High NPR was associated with the increased risk of HT especially PH in patients with acute ischemic stroke.

Keywords: neutrophil-to-platelet ratio, stroke, hemorrhagic transformation, parenchymal hematoma, outcome

\section{INTRODUCTION}

Acute ischemic stroke is among the leading causes of mortality and long-term morbidity throughout the world (1). Hemorrhagic transformation (HT) is a common and serious complication after acute ischemic stroke (2). Patients with HT were vulnerable to experience neurological deterioration, worse functional outcome and increased mortality (3). Moreover, HT is categorized into parenchymal hematoma $(\mathrm{PH})$ and hemorrhagic infarction (HI) based on the radiological appearance (4). A large population-based prospective study revealed that $\mathrm{PH}$ rather than HI was an independent risk factor for death or disability (5). Therefore, early recognition of $\mathrm{HT}$ especially $\mathrm{PH}$ is essential for the appropriate management and better prognosis in patients with acute ischemic stroke. 
Neutrophil, the key role in the innate immune response, has been found to be associated with ischemic stroke and HT $(6,7)$. One study comparing the prognostic value of different inflammatory factors indicated that neutrophil was a more sensitive indicator for cardiovascular mortality compared with other subsets of leukocyte and C-reactive protein (8). The activation of neutrophil after stroke could contribute to large infarct volume, blood-brain barrier (BBB) disruption and HT (9). Higher counts of neutrophil after ischemic stroke were associated with symptomatic intracranial hemorrhage and worse functional outcome (10). Moreover, the reduction in brain neutrophil recruitment through the inhibition of nod-like receptor protein 3 (NLRP3) could preserve the integrity of $\mathrm{BBB}$ and attenuate HT (11).

Platelet has been considered as a necessary factor against HT following ischemia/reperfusion because of its hemostatic function (12). The decrease in platelet count after ischemic stroke, caused by hemodilution of excessive fluid replacement (13), was correlated with the high rates of HT and even symptomatic HT $(14,15)$. Except for its hemostatic function, platelet also plays an important role in the inflammatory response (16). The interaction between neutrophil and platelet was involved in the process of vascular injury after ischemic stroke (16). Platelet could facilitate neutrophil recruitment and extravasation into brain parenchyma after stroke, and the amount of infiltrated neutrophil was correlated with stroke progression $(17,18)$. Moreover, high neutrophil-platelet complex formation may increase the risk of stroke in patients with symptomatic carotid stenosis (19). Several studies found that the activation and aggregation of neutrophil and platelet within cerebral microvessels resulted in vascular inflammation and BBB dysfunction following ischemic-reperfusion injury $(20,21)$.

Increased neutrophil count and decreased platelet count may be associated with poor functional outcome in patients with HT $(22,23)$. A recent study found that high neutrophil-to-platelet ratio (NPR) was associated with long-term poor outcome in patients with acute ischemic stroke (24). Indeed, NPR shows its advantage in revealing information about the crosstalk between inflammation and hemostasis, and has been suggested as a useful and rapid screening tool to assess systemic inflammation in infective endocarditis (25). However, to date, no study has investigated the relationship between NPR and HT after ischemic stroke. The present study was designed to explore whether high NPR was associated with HT especially PH in patients with acute ischemic stroke.

\section{MATERIALS AND METHODS}

\section{Subjects}

This was a retrospective study of HT patients who had been consecutively admitted to the Stroke Unit at the First Affiliated Hospital of Wenzhou Medical University between October 2011 and September 2018. The exclusion criteria were as follows: (1) acute infection within 2 weeks before admission or chronic infection; and (2) cancer, severe hepatic or renal diseases. Meanwhile, 270 age- and gender-matched controls without HT were included for comparison.
The study was approved by the ethics committee of the First Affiliated Hospital of Wenzhou Medical University, and the protocol followed the local ethics criteria for human research. Although written informed consent was not obtained for this study because of the retrospective design, it was obtained for data collection from our stroke registry.

\section{Clinical and Radiological Variables}

Data were collected containing demographics (age and gender), risk factors (hypertension, diabetes mellitus, coronary heart disease, atrial fibrillation, history of stroke, and cigarette smoking), systolic blood pressure, diastolic blood pressure, the Trial of ORG10172 in the Acute Stroke Treatment (TOAST) classification and treatment in hospital (thrombolysis, antiplatelet and anticoagulation). The stroke severity was assessed by the National Institutes of Health Stroke Scale (NIHSS) at admission (26). Functional outcome was assessed by the Barthel Index (BI) at discharge, and the BI $<60$ was defined as poor functional outcome (27).

All patients underwent brain Computed Tomography (CT)/Magnetic Resonance Imaging (MRI) scans at admission, at day $4( \pm 2)$ and at any clinical worsening. HT was diagnosed using follow-up CT/MRI scans, and classified into $\mathrm{PH}$ and $\mathrm{HI}$ according to the recommendations of European Cooperative Acute Stroke Study (ECASS) II classification (28). PH was defined as hemorrhage with a mass effect. HT was determined separately by two neurologists blinded to clinical data, and the third was consulted when a divergence occurred. Moreover, the infarct location was divided into two groups: anterior circulation included frontal, parietal, lateral temporal cortical and subcortical regions, internal capsule, and basal ganglia; posterior circulation included brainstem, cerebellum, thalamus, medial temporal, and occipital regions (29). The infarct size was categorized as follows: less than one-half of a lobe was classified as small infarct volume, and more than one-half of a lobe was classified as large infarct volume (30).

\section{Laboratory Test}

Blood samples were collected at admission in the Department of Emergency of our hospital and were obtained from the antecubital vein. The counts of leukocyte, neutrophil, lymphocyte, monocyte and platelet were obtained. The NPR was calculated by neutrophil count $\left(\times 10^{9} \mathrm{~L}\right) \times 1000 /$ platelet count $\left(\times 10^{9} / \mathrm{L}\right)$. NPR was further divided into tertiles (tertile 1 , tertile 2, tertile 3 ) in all patients and HT patients, respectively. The leukocyte-platelet ratio was calculated by leukocyte count $\left(\times 10^{9} \mathrm{~L}\right) \times 1000 /$ platelet count $\left(\times 10^{9} / \mathrm{L}\right)$. The lymphocyteplatelet ratio was calculated by lymphocyte count $\left(\times 10^{9} \mathrm{~L}\right)$ $\times 1000 /$ platelet count $\left(\times 10^{9} / \mathrm{L}\right)$. The monocyte-platelet ratio was calculated by monocyte count $\left(\times 10^{9} \mathrm{~L}\right) \times 1000 /$ platelet count $\left(\times 10^{9} / \mathrm{L}\right)$.

\section{Statistical Analysis}

All patients were divided into HT and non-HT, and the HT group was further divided into $\mathrm{PH}$ and $\mathrm{HI}$. The data were displayed as mean (standard deviation, SD) or median (interquartile range, IQR) for the continuous variables and percentages for 
the categorical variables. Student's $t$-test, analysis of variance (ANOVA) or Mann-Whitney U-test were applied for continuous variables, while the Chi-squared test was applied for proportions. On the one hand, receiver-operating characteristic (ROC) curves analysis were used to determine diagnostic accuracy of HT, and the cut-off values of NPR were calculated according to the Youden index. The area under the ROC curve (AUC) was considered as a critical diagnostic index. One the other hand, the levels of NPR were analyzed according to the degree of HT, while the degree of HT was also compared according to the NPR tertiles. Furthermore, multiple logistic regression analysis was used to evaluate whether NPR was associated with the incidence of HT and PH. For HT, model 1 was adjusted for age and sex; model 2 was adjusted for the variables in model 1 plus the factors that had already been established as predictors of HT (diabetes mellitus, atrial fibrillation, systolic blood pressure, large infarct volume, baseline NIHSS, anticoagulant and thrombolysis); and model 3 was adjusted for all the variables in model 2 plus the factors that significantly differed between the HT groups on the univariate analysis (coronary heart disease, anterior circulation and antiplatelet). For $\mathrm{PH}$, model 1 was adjusted for age and sex; and model 2 was adjusted for the variables in model 1 plus the factors that had already been established as predictors of HT and that significantly differed between the outcome groups on the univariate analysis (diabetes mellitus, atrial fibrillation, systolic blood pressure, large infarct volume, baseline NIHSS, anticoagulant and thrombolysis). Odds ratios (ORs) and 95\% confidence intervals (CIs) were calculated. $P<0.05$ at two-tailed was considered statistically significant. All statistical analyses were performed on SPSS for Windows, version 23.0 (SPSS Inc., Chicago, IL, USA).

\section{RESULTS}

\section{Characteristics of Patients With HT/PH}

This study enrolled 549 patients: 375 men (68.3\%) and 174 women $(31.7 \%)$. Their mean age was $69.0 \pm 12.3$ years. Among the 279 patients with HT, 131 patients had $\mathrm{PH}$ and 148 patients

TABLE 1 | Differences of the characteristics according to the subcategorized groups of HT.

\begin{tabular}{|c|c|c|c|c|c|c|}
\hline & \multicolumn{3}{|c|}{ All patients $(n=549)$} & \multicolumn{3}{|c|}{ Patients with HT ( $n=279$ ) } \\
\hline \multicolumn{7}{|l|}{ Demographics } \\
\hline Age $(y)$, mean $\pm S D$ & $69.1 \pm 12.1$ & $68.9 \pm 12.6$ & 0.815 & $69.3 \pm 11.8$ & $68.4 \pm 13.4$ & 0.548 \\
\hline Male, $n(\%)$ & $181(67.0)$ & $85(30.5)$ & 0.530 & $103(69.6)$ & $91(69.5)$ & 0.981 \\
\hline Diabetes mellitus & $75(27.8)$ & $69(24.7)$ & 0.417 & 35 (23.6) & $34(26.0)$ & 0.656 \\
\hline Coronary heart disease & $14(5.2)$ & $32(11.5)$ & $0.008^{*}$ & $18(12.2)$ & $14(10.7)$ & 0.700 \\
\hline Atrial fibrillation & $26(9.7)$ & $104(37.3)$ & $<0.001^{\star \star}$ & $43(29.1)$ & $61(46.6)$ & $0.003^{\star}$ \\
\hline History of stroke & $31(11.5)$ & $40(14.3)$ & 0.319 & 25 (16.9) & $15(11.5)$ & 0.196 \\
\hline Cigarette smoking & $115(42.6)$ & $122(43.7)$ & 0.788 & $68(45.9)$ & $54(41.2)$ & 0.427 \\
\hline Stroke mechanisms, $n$ (\%) & & & $<0.001^{\star \star}$ & & & $0.002^{*}$ \\
\hline Large-artery atherosclerosis & $245(90.7)$ & $157(56.3)$ & & $97(65.5)$ & $60(45.8)$ & \\
\hline Cardioembolism & $20(7.4)$ & $118(42.3)$ & & $49(33.1)$ & $69(52.7)$ & \\
\hline Others & $5(1.9)$ & $4(1.4)$ & & $2(1.4)$ & $2(1.5)$ & \\
\hline \multicolumn{7}{|l|}{ Infarct location, $n$ (\%) } \\
\hline Anterior circulation & $183(67.8)$ & $257(92.1)$ & $<0.001^{\star \star}$ & $133(89.9)$ & $124(94.7)$ & 0.138 \\
\hline Posterior circulation & $126(46.7)$ & $117(41.9)$ & 0.265 & $68(45.9)$ & $49(37.4)$ & 0.149 \\
\hline Large infarct volume, n (\%) & $5(1.9)$ & $117(41.9)$ & $<0.001^{\star \star}$ & $51(34.5)$ & $66(50.4)$ & $0.007^{\star}$ \\
\hline \multicolumn{7}{|l|}{ Treatment in hospital, $n(\%)$} \\
\hline Thrombolysis & $5(1.9)$ & $20(7.2)$ & $0.003^{\star}$ & $7(4.7)$ & $13(9.9)$ & 0.093 \\
\hline
\end{tabular}

$H T$, hemorrhagic transformation; HI, hemorrhagic infarct; PH, parenchymal hematoma; SD, standard deviation; SBP, systolic blood pressure; DBP, diastolic blood pressure; IQR, interquartile range; NPR, neutrophil-to-platelet ratio; ${ }^{*} P<0.05 ;{ }^{* *} P<0.001$. 


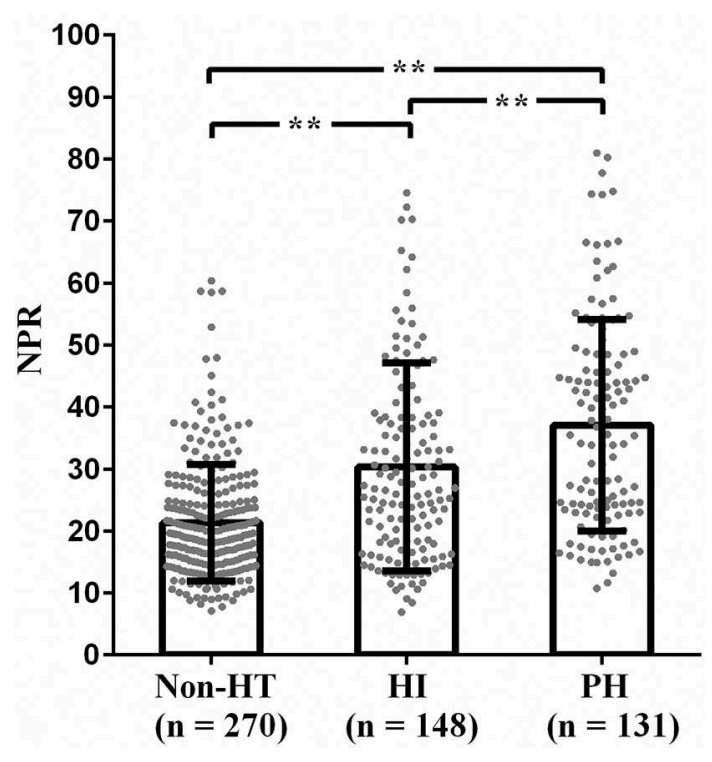

FIGURE 1 | The levels of NPR in the subcategorized groups of HT. (1) The NPR was higher in patients with $\mathrm{H}$ than those with non-HT [26.6 (17.9-38.3) vs. 19.1 (14.8-24.8), $P<0.001$ ]; (2) The NPR was higher in patients with $\mathrm{PH}$ than those with non-HT [36.8 (23.7-49.2) vs. 19.1 (14.8-24.8), $P<0.001]$; (3) The NPR was higher in patients with $\mathrm{PH}$ than those with $\mathrm{HI}$ [36.8 (23.7-49.2) vs. 26.6 (17.9-38.3)], $P<0.001$ ). NPR, neutrophil-to-platelet ratio; HT, hemorrhagic transformation; HI, hemorrhagic infarct; $\mathrm{PH}$, parenchymal hematoma; ${ }^{\star \star} P<0.001$

had HI. Clinical characteristics in patients with $\mathrm{HT} / \mathrm{PH}$ and those without are summarized in Table 1. Patients with HT/PH were more likely to have atrial fibrillation, high baseline NIHSS and large infarct volume. The therapy of thrombolysis, anticoagulant and antiplatelet were more frequently observed in patients with HT than those without HT. Patients with $\mathrm{PH}$ had higher proportion of poor functional outcome compared with those with HI.

Neutrophil count was significantly higher in the patients with $\mathrm{PH}$ than those with HI or non-HT [7.0 \pm 3.6 vs. $5.7 \pm$ 3.0 vs. $4.2 \pm 1.5, P<0.001]$. Platelet count was significantly lower in the patients with $\mathrm{PH}$ than those with HI or nonHT $[190.3 \pm 63.2$ vs. $205.8 \pm 70.2$ vs. $207.0 \pm 51.2, P=$ 0.025]. According to the ROC curves, with an AUC of 0.733, NPR showed a greater discriminatory ability compared with neutrophil count [AUC 0.727, 95\% CI (0.685-0.769), $P<0.001$ ] and platelet count [AUC 0.570, 95\% CI (0.521-0.617), $P=$ 0.005] (Supplementary Figure 1). With an AUC of 0.733, NPR also showed a greater discriminatory ability compared with leukocyte-platelet ratio [AUC 0.695, 95\% CI (0.651-0.740), $P<$ 0.001 ], lymphocyte-platelet ratio [AUC 0.355 , 95\% CI $(0.308-$ $0.401), P<0.001$ ], and monocyte-platelet ratio [AUC 0.664, 95\% CI (0.618-0.710), $P<0.001$ ] (Supplementary Figure 2).

\section{Association Between NPR and HT/PH}

NPR was significantly higher in the patients with $\mathrm{PH}$ than those with HI or non-HT [36.8 (23.7-49.2) vs. 26.6 (17.9-38.3) vs. 19.1
(14.8-24.8), $P<0.001$ ] (Figure 1). Furthermore, all patients were divided into three subgroups according to tertiles of NPR levels (tertile 1, <18.4; tertile 2, 18.4-29.1; and tertile 3, > 29.1). Patients with high NPR had a higher incidence of HT compared with those with middle or low NPR, respectively (77.2\% vs. $42.3 \%$ vs. $32.8 \% ; P<0.001$ ) (Figure $2 \mathrm{~A}$ ). With all patients taken as a whole, the incidence of HT taken as a dependent variable and low NPR taken as the reference used for NPR in the logistic analysis. Highest NPR was associated with the incidence of HT after adjustment for variables with clinical significance (model 2: OR = $2.503,95 \%$ CI: $1.381-4.537, P=0.002)$. The association remained significant after adjusting for factors that significantly differed between the HT groups on the univariate analysis (model 3: OR $=2.000,95 \%$ CI: $1.041-3.843, P=0.037$ ). Atrial fibrillation, large infarct volume, baseline NIHSS and anterior circulation were also risk factors of HT (Table 2). According to the ROC curves, the optimal cut-off value for NPR as a diagnostic marker of HT was 24.24 , with a sensitivity of $65.9 \%$ and a specificity of $72.6 \%$ [AUC 0.733 , 95\% CI (0.691-0.775), $P<0.001$ ] (Figure 3). The NPR above the cut-off (24.24) remained independently associated with HT after adjustment for confounding variables (OR 2.069, 95\% CI 1.195-3.584, $P=0.009$ ).

When the HT patients were classified into tertiles of NPR levels (tertile 1, <23.9; tertile 2, 23.9-39.9; and tertile 3, > 39.9), HT patients with high NPR had a higher incidence of $\mathrm{PH}$ compared with those with middle or low NPR, respectively (66.0\% vs. $39.1 \%$ vs. $35.5 \% ; P<0.001)$ (Figure 2B). With $\mathrm{HT}$ patients taken as a whole, the incidence of $\mathrm{PH}$ taken as a dependent variable and low NPR taken as the reference used for NPR in the logistic analysis. Highest NPR remained independently associated with the incidence of $\mathrm{PH}$ (model 2: OR $=2.641$, 95\% CI: $1.308-5.342, P=0.007$ ), after adjustment for confounding factors. Baseline NIHSS was also a risk factor of $\mathrm{PH}$ (Table 3). According to the ROC curve, the optimal cut-off value for NPR as a diagnostic marker of $\mathrm{PH}$ was 39.40 , with a sensitivity of $48.1 \%$ and a specificity of $78.4 \%$ [AUC $0.641,95 \%$ CI $(0.577-$ 0.706), $P<0.001$ ] (Figure 3). The NPR above the cut-off (39.40) remained independently associated with $\mathrm{PH}$ after adjustment for confounding variables (OR 2.724, 95\% CI 1.497-4.959, $P=$ 0.001). Moreover, similar conclusion was attained when the NPR level was continuous variable in the logistic regression analysis [HT $(\mathrm{OR}=1.03395 \% \mathrm{CI}, 1.009-1.057, P=0.006)$ and PH (OR $=1.020,95 \% \mathrm{CI}, 1.004-1.036, P=0.013)]$.

\section{DISCUSSION}

To the best of our knowledge, this is the first study to explore the association between NPR and HT in patients with acute ischemic stroke. The present study indicated that high NPR was associated with an increased risk of HT after acute ischemic stroke. Moreover, we also found that HT patients with higher NPR were more likely to develop PH.

Accumulating studies have indicated that HT was correlated with ischemia/reperfusion injury, which was mainly attributed to $\mathrm{BBB}$ disruption, hemostatic dysfunction, oxidative stress and inflammation (31). One study investigating the relationship 

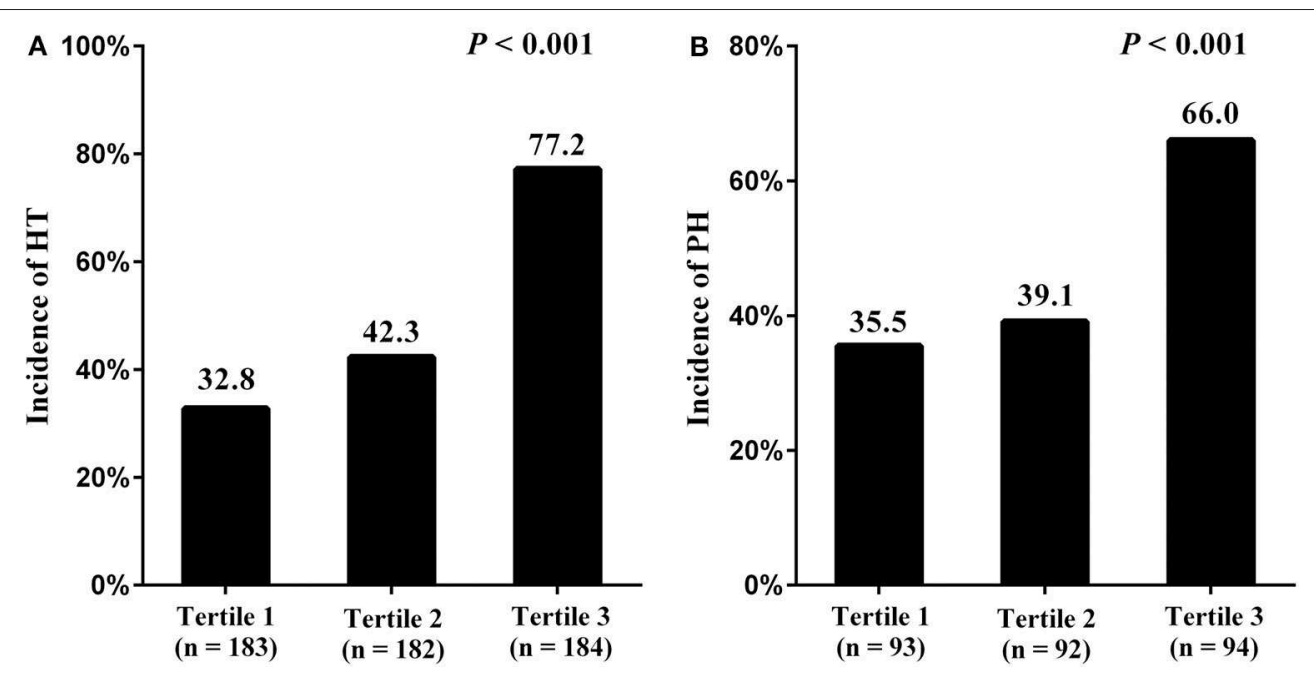

FIGURE 2 | Incidence of HT and PH stratified by NPR tertiles [NPR tertiles in all patients: tertile 1, <18.4; tertile 2, 18.4-29.1; tertile 3, >29.1 (A). NPR tertiles in patients with HT: tertile 1, <23.9; tertile 2, 23.9-39.9; tertile 3, >39.9 (B)]. NPR, neutrophil-to-platelet ratio; HT, hemorrhagic transformation; PH, parenchymal hematoma.

TABLE 2 | Factors associated with HT by multivariate logistic regression analysis.

\begin{tabular}{|c|c|c|c|c|c|c|}
\hline & \multicolumn{2}{|c|}{ Model 1} & \multicolumn{2}{|c|}{ Model 2} & \multicolumn{2}{|l|}{ Model 3} \\
\hline & OR $(95 \% \mathrm{Cl})$ & $P$-value & OR (95\% Cl) & $P$-value & OR (95\% Cl) & $P$-value \\
\hline \multicolumn{7}{|l|}{ NPR } \\
\hline Tertile 1 & Reference & & Reference & & Reference & \\
\hline Tertile 2 & $1.521(0.991-2.335)$ & 0.055 & $0.946(1.299-1.812)$ & 0.837 & $1.017(0.575-1.800)$ & 0.953 \\
\hline Tertile 3 & $7.245(4.525-11.600)$ & $<0.001$ & $7.839(2.842-4.301)$ & 0.005 & $2.000(1.041-3.843)$ & 0.037 \\
\hline Atrial fibrillation & & & $2.627(1.353-5.102)$ & 0.004 & $2.514(1.242-5.089)$ & 0.010 \\
\hline Large infarct volume & & & 7.839 (2.842-21.625) & $<0.001$ & 4.810 (1.679-13.780) & 0.003 \\
\hline Baseline NIHSS & & & 1.249 (1.175-1.329) & $<0.001$ & $1.256(1.176-1.343)$ & $<0.001$ \\
\hline Anterior circulation & & & & & $4.133(2.068-8.261)$ & $<0.001$ \\
\hline Antiplatelet & & & & & $0.213(0.109-0.417)$ & $<0.001$ \\
\hline
\end{tabular}

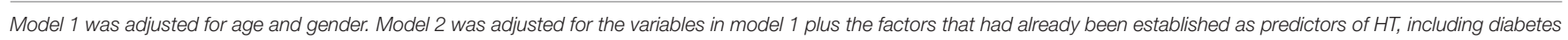

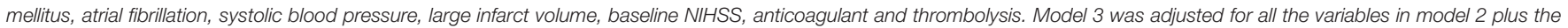

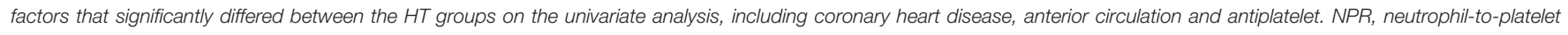
ratio; HT, hemorrhagic transformation; OR, odd ratio; Cl, confidence interval; NIHSS, National Institutes of Health Stroke Scale.

between $\mathrm{BBB}$ permeability and degree of HT found that higher $B B B$ permeability may contribute to major intracranial bleeding in patients with acute ischemic stroke (32). Another neuroimaging study found that mild $\mathrm{BBB}$ disruption in ischemic brain was reversible due to the early reperfusion, while severe BBB disruption after sustained ischemia may increase the risk of $\mathrm{HT}$ and $\mathrm{PH}$ (33). Moreover, the increase in matrix metalloproteinase-9 (MMP-9) during the inflammatory phase of ischemic stroke was related to HT, and a $24 \mathrm{~h}$ peak of MMP-9 occurred before PH (34). The inhibition of MMP9 expression with baicalin could maintain the BBB integrity, attenuate thrombolysis-induced HT and improve the prognosis in patients with acute ischemic stroke (35).

Several studies have indicated that neutrophil played an important role in the BBB disruption after stroke $(22,36)$. Neutrophils could release pro-inflammatory factors, reactive oxygen species and proteolytic enzymes, leading to BBB disruption and brain injury $(9,37)$. Previous studies found that neutrophil was the major source of MMP-9 acting on the BBB, which may result in symptomatic HT and poor outcome after ischemic stroke $(10,38)$. Higher neutrophil count was correlated with higher intracerebral hemorrhage volume at admission (39). The suppression of neutrophil recruitment was found to reduce hemorrhage volume and attenuate the severity of HT (11). While one study showed that low neutrophil count was associated with an increased risk of hematoma expansion during the hyperacute phase of intracerebral hemorrhage (40). One explanation for the contradictory findings may be that the effect of neutrophil on the vascular injury may be mediated by platelet, and the role of neutrophil-platelet interaction in the vascular inflammation may be varied during the different phase of intracerebral hemorrhage (41). The interaction between neutrophil and platelet could enhance the reactive oxygen species generation and exasperate the vascular injury (42), despite of the procoagulant properties 

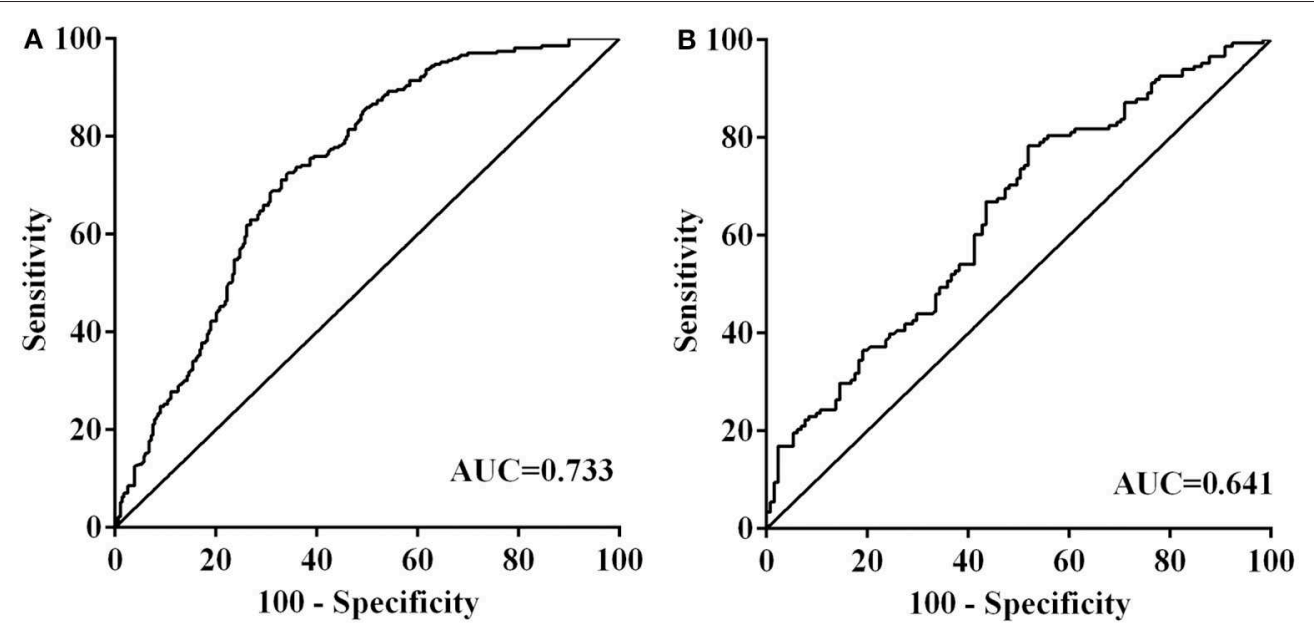

FIGURE 3 | Receiver operator characteristic analysis of neutrophil-to-platelet ratio for predicting hemorrhagic transformation (A) and parenchymal hematoma (B). AUC, area under the curve.

TABLE 3 | Factors associated with PH by multivariate logistic regression analysis.

\begin{tabular}{|c|c|c|c|c|}
\hline & \multicolumn{2}{|l|}{ Model 1} & \multicolumn{2}{|l|}{ Model 2} \\
\hline & OR $(95 \% \mathrm{Cl})$ & $\boldsymbol{P}$-value & OR $(95 \% \mathrm{Cl})$ & $P$-value \\
\hline \multicolumn{5}{|l|}{ NPR } \\
\hline Tertile 1 & Reference & & Reference & \\
\hline Tertile 2 & 1.249 (0.680-2.293) & 0.474 & $0.975(0.512-1.855)$ & 0.938 \\
\hline Tertile 3 & $3.971(2.121-7.437)$ & $<0.001$ & $2.641(1.308-5.342)$ & 0.007 \\
\hline Atrial fibrillation & & & $1.492(0.844-2.638)$ & 0.169 \\
\hline Large infarct volume & & & $1.073(0.607-1.899)$ & 0.808 \\
\hline Baseline NIHSS & & & $1.074(1.016-1.135)$ & 0.011 \\
\hline
\end{tabular}

Model 1 was adjusted for age and gender. Model 2 was adjusted for the variables in model 1 plus the factors that had already been established as predictors of HT and that significantly differed between the outcome groups on the univariate analysis, including diabetes mellitus, atrial fibrillation, systolic blood pressure, large infarct volume, baseline NIHSS, anticoagulant and thrombolysis. NPR, neutrophil-to-platelet ratio; PH, parenchymal hematoma; OR, odd ratio; Cl, confidence interval; NIHSS, National Institutes of Health Stroke Scale.

of the activated neutrophils $(43,44)$. Moreover, platelet serves as a major contributor of several pro-inflammatory factors like tumor necrosis factor- $\alpha$ (41), which contributed to the increase in activated neutrophils and neutrophil-platelet aggregates (45). An animal study found that the depletion of platelet reduced the neutrophil recruitment and vascular inflammation after the occlusion of the middle cerebral artery (46).

In addition, a review of neutrophil-platelet interactions revealed that activated platelets were involved in the release of inflammatory mediators, neutrophil accumulation and the increase in vascular permeability (16). Platelet-endothelial interactions might prevent or heal neutrophil-induced vascular injury through the local release of soluble vasculoprotective factors (47). Hemostatic function of platelets was also attributed to the formation of plugs and clotting at the site of vessel injury (48), which helped to maintain the BBB integrity (49). Systemic inflammation was often accompanied by low platelet count, and consumption of platelet may be caused by the immunological process in the circulation (41). Therefore, it can be inferred that patients with higher NPR may have more severe disruption of $\mathrm{BBB}$, leading to higher incidence of $\mathrm{HT}$ and $\mathrm{PH}$. The better comprehension of neutrophil-platelet interactions may pave the way to promote neuroprotection and vascular repair in response to systematic inflammation after ischemic stroke (16).

In the present study, patients with $\mathrm{PH}$ had greater stroke severity and worse functional outcome compared with those with HI. A recent study supporting our findings showed that $\mathrm{PH}$ rather than $\mathrm{HI}$ was independently associated with poor functional outcome after thrombectomy in patients with acute large vessel occlusion (50). Some studies even indicated that patients with HI had a better functional outcome than those without HT because HI was a sign of early revascularization and better reperfusion $(51,52)$. Consistent with previous studies, we found that atrial fibrillation and large infarct volume were risk factors of HT (2). Moreover, we also found that anterior circulation was associated with HT. One possible reason for this phenomenon may be that patients with anterior circulation stroke were more likely to have greater stroke severity, atrial fibrillation and vessel occlusion, which were correlated with HT $(2,53)$.

There are some limitations in this study. First, the NPR was recorded only once, and it is necessary to investigate the association of dynamic changes in NPR after stroke with HT. Second, we did not explore the mechanisms underlying the influence of neutrophil and platelet on the BBB disruption in animal models, which should be conducted in our future work. Third, the functional outcome in stroke patients was assessed by BI, and our prospective studies will be conducted to better assess the functional outcome by modified Rankin scale (mRS). Fourth, the infarct size was taken as categorical variables in the analysis, and it is better to estimate the infarct size using the Alberta Stroke Program Early CT Score (ASPECTS) system by trained radiologists. Finally, considering the role of inflammation in the HT is still complex, more studies should be taken to further and better record more inflammatory index in the future. 


\section{CONCLUSIONS}

The present study demonstrated that high NPR was associated with the risk of $\mathrm{HT}$ and $\mathrm{PH}$ in patients with acute ischemic stroke. These findings may help clinicians to identify which stroke patients are at high risk of $\mathrm{HT}$ especially $\mathrm{PH}$, and thus conduct appropriate therapy and CT scan in this population. Considering the limitations in the present study, this relationship needs further investigations in future.

\section{DATA AVAILABILITY STATEMENT}

The raw data supporting the conclusions of this manuscript will be made available by the corresponding author, without undue reservation, to any qualified researcher upon reasonable request.

\section{ETHICS STATEMENT}

The studies involving human participants were reviewed and approved by The First Affiliated Hospital of Wenzhou Medical University. Written informed consent for participation was not required for this study in accordance with the national legislation and the institutional requirements.

\section{REFERENCES}

1. Hasan TF, Rabinstein AA, Middlebrooks EH, Haranhalli N, Silliman SL, Meschia JF, et al. Diagnosis and management of acute ischemic stroke. Mayo Clin Proc. (2018) 93:523-38. doi: 10.1016/j.mayocp.2018.02.013

2. Jickling GC, Liu DZ, Stamova B, Ander BP, Zhan XH, Lu AG, et al. Hemorrhagic transformation after ischemic stroke in animals and humans. J Cereb Blood Flow Metab. (2014) 34:185-99. doi: 10.1038/jcbfm.2013.203

3. Yaghi S, Willey JZ, Cucchiara B, Goldstein JN, Gonzales NR, Khatri P, et al. Treatment and outcome of hemorrhagic transformation after intravenous alteplase in acute ischemic stroke: a scientific statement for healthcare professionals from the American Heart Association/American Stroke Association. Stroke. (2017) 48:E343-61. doi: 10.1161/STR.0000000000000152

4. Guenego A, Lecler A, Raymond J, Sabben C, Khoury N, Premat K, et al. Hemorrhagic transformation after stroke: inter- and intrarater agreement. Eur J Neurol. (2019) 26:476-82. doi: 10.1111/ene.13859

5. Paciaroni M, Agnelli G, Corea F, Ageno W, Alberti A, Lanari A, et al. Early hemorrhagic transformation of brain infarction: rate, predictive factors, and influence on clinical outcome - results of a prospective multicenter study. Stroke. (2008) 39:2249-56. doi: 10.1161/STROKEAHA.107.510321

6. Bonaventura A, Liberale L, Vecchie A, Casula M, Carbone F, Dallegri F, et al. Update on inflammatory biomarkers and treatments in ischemic stroke. Int J Mol Sci. (2016) 17:53. doi: 10.3390/ijms17121967

7. Ritzel RM, Lai YJ, Crapser JD, Patel AR, Schrecengost A, Grenier JM, et al. Aging alters the immunological response to ischemic stroke. Acta Neuropathol. (2018) 136:89-110. doi: 10.1007/s00401-018-1859-2

8. Hartaigh BO, Bosch JA, Thomas GN, Lord JM, Pilz S, Loerbroks A, et al. Which leukocyte subsets predict cardiovascular mortality? From the LUdwigshafen RIsk and Cardiovascular Health (LURIC) Study. Atherosclerosis. (2012) 224:161-9. doi: 10.1016/j.atherosclerosis.2012.04.012

9. Jickling GC, Liu DZ, Ander BP, Stamova B, Zhan XH, Sharp FR. Targeting neutrophils in ischemic stroke: translational insights from experimental studies. J Cereb Blood Flow Metab. (2015) 35:888-901. doi: $10.1038 /$ jcbfm.2015.45

10. Maestrini I, Strbian D, Gautier S, Haapaniemi E, Moulin S, Sairanen $\mathrm{T}$, et al. Higher neutrophil counts before thrombolysis for cerebral

\section{AUTHOR CONTRIBUTIONS}

$\mathrm{WH}$ and $\mathrm{JH}$ designed the project. WH did the statistical analyses and wrote the manuscript draft. WH, YR, CY, QC, HCheng, YZ, YC, GH, and HChen screened and extracted data. All authors have made an intellectual contribution to the manuscript and approved the submission.

\section{FUNDING}

This work was supported by the Projects of National Science Foundation of China (No.81873799), the National Key Technology Research and Development Program of the Ministry of Science and Technology of China (grant number: 2015BAI13B01) and Wenzhou Municipal Sci-Tech Bureau Program (Y20160002). These sources had no further role in study design, data collection and analysis, decision to publish, or preparation of the article.

\section{SUPPLEMENTARY MATERIAL}

The Supplementary Material for this article can be found online at: https://www.frontiersin.org/articles/10.3389/fneur. 2019.01310/full\#supplementary-material

ischemia predict worse outcomes. Neurology. (2015) 85:1408-16. doi: 10.1212/WNL.0000000000002029

11. Guo ZL, Yu SH, Chen X, Zheng P, Hu T, Duan ZH, et al. Suppression of NLRP3 attenuates hemorrhagic transformation after delayed rtPA treatment in thromboembolic stroke rats: involvement of neutrophil recruitment. Brain Res Bull. (2018) 137:229-40. doi: 10.1016/j.brainresbull.2017.12.009

12. Stone JA, Willey JZ, Keyrouz S, Butera J, McTaggart RA, Cutting S, et al. Therapies for hemorrhagic transformation in acute ischemic stroke. Curr Treat Options Neurol. (2017) 19:15. doi: 10.1007/s11940-017-0438-5

13. Woessner R, Grauer MT, Dieterich HJ, Treib W, Stoll M, Treib J. Influence of long-term volume therapy with hydroxyethyl starch on leukocytes in patients with acute stroke. Arzneimittelforschung. (2003) 53:402-6. doi: 10.1055/s-0031-1297127

14. Kidwell CS, Saver JL, Carneado J, Sayre J, Starkman S, Duckwiler G, et al. Predictors of hemorrhagic transformation in patients receiving intra-arterial thrombolysis. Stroke. (2002) 33:717-24. doi: 10.1161/hs0302.104110

15. Lee JH, Park KY, Shin JH, Cha JK, Kim HY, Kwon JH, et al. Symptomatic hemorrhagic transformation and its predictors in acute ischemic stroke with atrial fibrillation. Eur Neurol. (2010) 64:193-200. doi: 10.1159/000319048

16. Garcia-Culebras A, Duran-Laforet V, Pena-Martinez C, Ballesteros I, Pradillo JM, Diaz-Guzman J, et al. Myeloid cells as therapeutic targets in neuroinflammation after stroke: specific roles of neutrophils and neutrophil-platelet interactions. J Cereb Blood Flow Metab. (2018) 38:2150-64. doi: 10.1177/0271678X18795789

17. Tang CM, Wang C, Zhang Y, Xue LJ, Li YY, Ju CY, et al. Recognition, intervention, and monitoring of neutrophils in acute ischemic stroke. Nano Lett. (2019) 19:4470-7. doi: 10.1021/acs.nanolett.9b01282

18. Cuartero MI, Ballesteros I, Lizasoain I, Moro MA. Complexity of the cell-cell interactions in the innate immune response after cerebral ischemia. Brain Res. (2015) 1623:53-62. doi: 10.1016/j.brainres.2015.04.047

19. McCabe DJH, Harrison P, Mackie IJ, Sidhu PS, Lawrie AS, Watt H, et al. Increased platelet count and leucocyte-platelet complex formation in acute symptomatic compared with asymptomatic severe carotid stenosis. J Neurol Neurosurg Psychiatry. (2005) 76:1249-54. doi: 10.1136/jnnp.2004.051003

20. Vital SA, Becker F, Holloway PM, Russell J, Perretti M, Granger $\mathrm{DN}$, et al. Formyl-peptide receptor 2/3/lipoxin A(4) receptor regulates 
neutrophil-platelet aggregation and attenuates cerebral inflammation impact for therapy in cardiovascular disease. Circulation. (2016) 133:2169-79. doi: 10.1161/CIRCULATIONAHA.115.020633

21. Li G, Sanders JM, Bevard MH, Sun Z, Chumley JW, Galkina EV, et al. CD40 ligand promotes Mac-1 expression, leukocyte recruitment, and neointima formation after vascular injury. Am J Pathol. (2008) 172:1141-52. doi: 10.2353/ajpath.2008.070633

22. Rosell A, Cuadrado E, Ortega-Aznar A, Hernandez-Guillamon M, Lo EH, Montaner J. MMP-9-Positive neutrophil infiltration is associated to bloodbrain barrier breakdown and basal lamina type IV collagen degradation during hemorrhagic transformation after human ischemic stroke. Stroke. (2008) 39:1121-6. doi: 10.1161/STROKEAHA.107.500868

23. Jiao YG, Li GC, Xing YL, Nie DA, Liu XT. Influencing factors of hemorrhagic transformation in non-thrombolysis patients with cerebral infarction. Clin Neurol Neurosurg. (2019) 181:68-72. doi: 10.1016/j.clineuro.2019. 04.018

24. Jin PP, Li XM, Chen J, Zhang ZR, Hu WW, Chen LY, et al. Platelet-toneutrophil ratio is a prognostic marker for 90 -days outcome in acute ischemic stroke. J Clin Neurosci. (2019) 63:110-15. doi: 10.1016/j.jocn.2019.01.028

25. Wei XB, Liu YH, He PC, Yu DQ, Tan N, Zhou YL, et al. The impact of admission neutrophil-to-platelet ratio on in-hospital and long-term mortality in patients with infective endocarditis. Clin Chem Lab Med. (2017) 55:899906. doi: 10.1515/cclm-2016-0527

26. Kim JT, Park MS, Choi KH, Kim BJ, Han MK, Park TH, et al. Clinical outcomes of posterior versus anterior circulation infarction with low National Institutes of Health stroke scale scores. Stroke. (2017) 48:55-62. doi: 10.1161/STROKEAHA.116.013432

27. Sulter G, Steen C, De Keyser J. Use of the Barthel Index and Modified Rankin Scale in acute stroke trials. Stroke. (1999) 30:1538-41. doi: 10.1161/01.STR.30.8.1538

28. Hacke W, Kaste M, Fieschi C, von Kummer R, Davalos A, Meier D, et al. Randomised double-blind placebo-controlled trial of thrombolytic therapy with intravenous alteplase in acute ischaemic stroke (ECASS II). Second European-Australasian Acute Stroke Study Investigators. Lancet. (1998) 352:1245-51. doi: 10.1016/S0140-6736(98)08020-9

29. Rovira A, Grive E, Rovira A, Alvarez-Sabin J. Distribution territories and causative mechanisms of ischemic stroke. Eur Radiol. (2005) 15:416-26. doi: 10.1007/s00330-004-2633-5

30. Brott T, Marler JR, Olinger CP, Adams HP Jr, Tomsick T, Barsan WG, et al. Measurements of acute cerebral infarction: lesion size by computed tomography. Stroke. (1989) 20:871-5. doi: 10.1161/01.STR.20.7.871

31. Lu GF, He QW, Shen Y, Cao F. Potential biomarkers for predicting hemorrhagic transformation of ischemic stroke. Int J Neurosci. (2018) 128:7989. doi: 10.1080/00207454.2017.1349766

32. Leigh R, Jen SS, Hillis AE, Krakauer JW, Barker PB, Investigators SVI. Pretreatment blood-brain barrier damage and post-treatment intracranial hemorrhage in patients receiving intravenous tissue-type plasminogen activator. Stroke. (2014) 45:2030-5. doi: 10.1161/STROKEAHA.114.005249

33. Simpkins AN, Dias C, Leigh R, National Institutes of Health Natural History of Stroke Investigators. Identification of reversible disruption of the human blood-brain barrier following acute ischemia. Stroke. (2016) 47:2405-8. doi: 10.1161/STROKEAHA.116.013805

34. Montaner J, Alvarez-Sabin J, Molina CA, Angles A, Abilleira S, Arenillas J, et al. Matrix metalloproteinase expression is related to hemorrhagic transformation after cardioembolic stroke. Stroke. (2001) 32:2762-7. doi: $10.1161 / \mathrm{hs} 1201.99512$

35. Chen HS, Guan BH, Chen X, Chen XM, Li CM, Qiu JH, et al. Baicalin attenuates blood-brain barrier disruption and hemorrhagic transformation and improves neurological outcome in ischemic stroke rats with delayed t-PA treatment: involvement of ONOO-MMP-9 pathway. Transl Stroke Res. (2018) 9:515-29. doi: 10.1007/s12975-017-0598-3

36. Dang QB, Lapergue B, Alexy TD, Diallo D, Moreno JA, Mazighi M, et al. High-density lipoproteins limit neutrophil-induced damage to the blood-brain barrier in vitro. J Cereb Blood Flow Metab. (2013) 33:575-82. doi: $10.1038 /$ jcbfm. 2012.206

37. Bechmann I, Galea I, Perry VH. What is the blood-brain barrier (not)? Trends Immunol. (2007) 28:5-11. doi: 10.1016/j.it.2006.11.007
38. Turner RJ, Sharp FR. Implications of MMP9 for blood brain barrier disruption and hemorrhagic transformation following ischemic stroke. Front Cell Neurosci. (2016) 10:13. doi: 10.3389/fncel.2016.00056

39. Adeoye O, Walsh K, Woo JG, Haverbusch M, Moomaw CJ, Broderick JP, et al. Peripheral monocyte count is associated with case fatality after intracerebral hemorrhage. J Stroke Cerebrovasc Dis. (2014) 23:E107-11. doi: 10.1016/j.jstrokecerebrovasdis.2013.09.006

40. Morotti A, Phuah CL, Anderson CD, Jessel MJ, Schwab K, Ayres AM, et al. Leukocyte count and intracerebral hemorrhage expansion. Stroke. (2016) 47:1473-8. doi: 10.1161/STROKEAHA.116.013176

41. Rossaint J, Margraf A, Zarbock A. Role of platelets in leukocyte recruitment and resolution of inflammation. Front Immunol. (2018) 9:13. doi: 10.3389/fimmu.2018.02712

42. Vanichakarn P, Blair P, Wu C, Freedman JE, Chakrabarti S. Neutrophil CD40 enhances platelet-mediated inflammation. Thromb Res. (2008) 122:346-58. doi: 10.1016/j.thromres.2007.12.019

43. McDonald B, Davis RP, Kim SJ, Tse M, Esmon CT, Kolaczkowska E, et al. Platelets and neutrophil extracellular traps collaborate to promote intravascular coagulation during sepsis in mice. Blood. (2017) 129:1357-67. doi: 10.1182/blood-2016-09-741298

44. Maugeri N, Baldini M, Ramirez GA, Rovere-Querini P, Manfredi AA. Plateletleukocyte deregulated interactions foster sterile inflammation and tissue damage in immune-mediated vessel diseases. Thromb Res. (2012) 129:267-73. doi: 10.1016/j.thromres.2011.12.001

45. Bhasym A, Annarapu GK, Saha S, Shrimali N, Gupta S, Seth T, et al. Neutrophils develop rapid proinflammatory response after engulfing Hbactivated platelets under intravascular hemolysis. Clin Exp Immunol. (2019) 197:131-40. doi: 10.1111/cei.13310

46. Sreeramkumar V, Adrover JM, Ballesteros I, Cuartero MI, Rossaint J, Bilbao I, et al. Neutrophils scan for activated platelets to initiate inflammation. Science. (2014) 346:1234-8. doi: 10.1126/science. 1256478

47. Ho-Tin-Noe B, Demers M, Wagner DD. How platelets safeguard vascular integrity. J Thromb Haemost. (2011) 9:56-65. doi: 10.1111/j.1538-7836.2011.04317.x

48. Stalker TJ, Welsh JD, Brass LF. Shaping the platelet response to vascular injury. Curr Opin Hematol. (2014) 21:410-17. doi: 10.1097/MOH.0000000000000070

49. Jones LD, Jackson JW, Maggirwar SB. Modeling HIV-1 induced neuroinflammation in mice: role of platelets in mediating blood-brain barrier dysfunction. PloS ONE. (2016) 11:26. doi: 10.1371/journal.pone.0151702

50. Lee YB, Yoon W, Lee YY, Kim SK, Baek BH, Kim JT, et al. Predictors and impact of hemorrhagic transformations after endovascular thrombectomy in patients with acute large vessel occlusions. J NeuroInterventional Surg. (2019) 11:469-73. doi: 10.1136/neurintsurg-2018-014080

51. Molina CA, Alvarez-Sabin J, Montaner J, Abilleira S, Arenillas JF, Coscojuela P, et al. Thrombolysis-related hemorrhagic infarction - a marker of early reperfusion, reduced infarct size, and improved outcome in patients with proximal middle cerebral artery occlusion. Stroke. (2002) 33:1551-6. doi: 10.1161/01.STR.0000016323.13456.E5

52. Kablau M, Kreisel SH, Sauer T, Binder J, Szabo K, Hennerici MG, et al. Predictors and early outcome of hemorrhagic transformation after acute ischemic stroke. Cerebrovasc Dis. (2011) 32:334-41. doi: 10.1159/000331702

53. De Marchis GM, Kohler A, Renz N, Arnold M, Mono ML, Jung S, et al. Posterior versus anterior circulation strokes: comparison of clinical, radiological and outcome characteristics. J Neurol Neurosurg Psychiatry. (2011) 82:33-7. doi: 10.1136/jnnp.2010.211151

Conflict of Interest: The authors declare that the research was conducted in the absence of any commercial or financial relationships that could be construed as a potential conflict of interest.

Copyright $\odot 2019$ He, Ruan, Yuan, Cheng, Cheng, Zeng, Chen, Huang, Chen and He. This is an open-access article distributed under the terms of the Creative Commons Attribution License (CC BY). The use, distribution or reproduction in other forums is permitted, provided the original author(s) and the copyright owner(s) are credited and that the original publication in this journal is cited, in accordance with accepted academic practice. No use, distribution or reproduction is permitted which does not comply with these terms. 